2.

\title{
Alther fezenprozente.
}

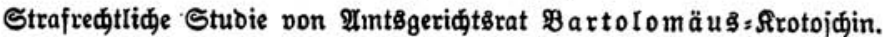

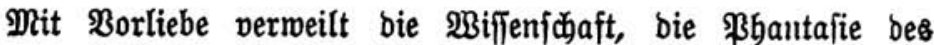
Menfojen bei benjenigen Ereigniffen ber Bsejdidte, bei benen in

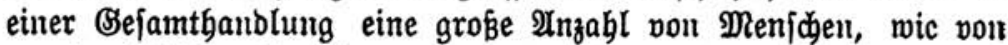
unmiderftehlidjer Bewalt getrieben, bem ßerberben anbeimfällt unter ber \$alto ibrer Bseguter.

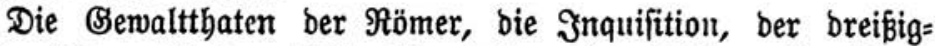

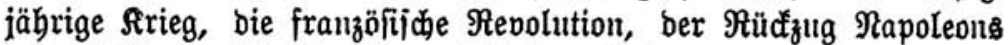

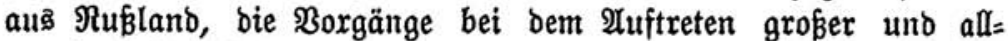
gemeiner Srantheiten find Stoffe, bie unaufgörlich bie (sejdidfts=

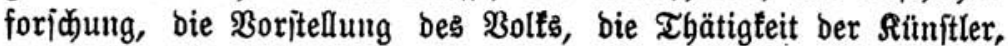
ber Didter befduäftigen. Es ift nidt zu viel gejagt, menn man behauptet, baß̧ gerabe bieje Ereigniffe biejentigen fint, bie aus ber

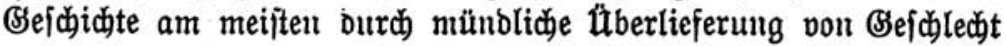

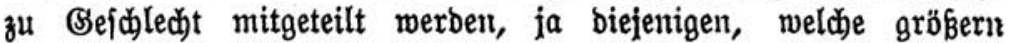

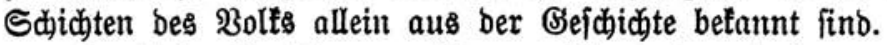

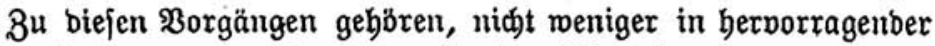
Weije, Die fogenannten Serenprozeffe, bas ßorgeben ber georbuteten Bsemalten ber ßölfer gegen ßerjonen, bie im Berbachte ftanden, über anbren unbefaunte Sräfte zum Sđjaben ber Menjobleit zu gebieten. Die Serenprozeffe gebören hauptjächlich unter jene all= gemeiner befaunten gefdidjtlichen Thatfachen, weil bas Jnterefie am Unntergange, an ber Bernichtung anbrer Mentiden bier noch vermebrt wird burch bas Jntereffe an bem 2Uukerorbentlichell, Beheimniszodlen Der Den Berfolgten und bem Untergange Serveihten

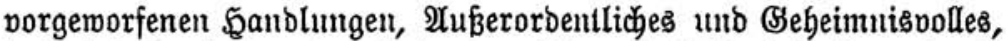
bas bei Taujenben burch bas unfichere (jefühl bejonbere Farbe

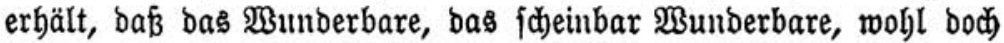




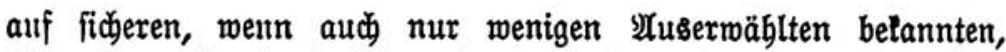
Brundlagen beruhe, baß bas Zaubern, Beifterbannen, ber Bertebr mit bem Teufel nicht jebem zuteil werben tönne, baß aber bod bies alles eine möglide, eine viel erftrebensosmerte Sacje jei.

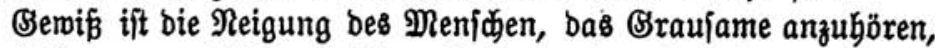
Bemaltthaten anzujehen, in feiner ఇeigung, bas Braujame zu thun, Bemalttbaten zu veritben, begründet, jobaß aljo beibes nur biejelbe

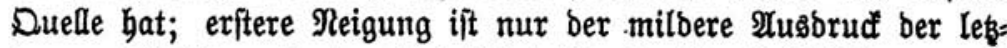

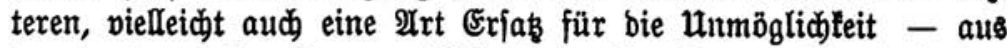
irgend einein Brrunbe - felbft etwas Braujames, Bemalttbätiges zul thun.

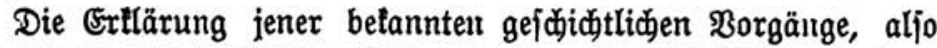
aud für bie Miöglichleit ber ŞerenprozefTe, wäre aljo leidit gefunben; aber, went fie riditig ift, wübe fie mur eine pindologifde $\mathrm{Er}=$ tlärung fein. Die Burzeln geididjtlicher Ereigniffe liegen aber nidit fo flar zut Tage und fint aud nidjt fo menige, baß fie mit eirtem Worte zu erflären wären; vielmebr reidjen fie, wie bie Er: eigniffe im Reben bes Einzelnen, nach allen Seiten uno in, oft nur

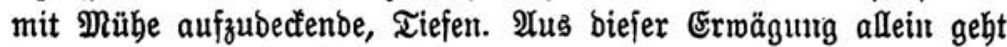
fdon hervor, baß geididitliche Ereignifie, jo wenig wie Ereignifje im Reben bes (sinzelnen, mit einer einfachen Rategorifierung $a b=$ zuthuu finto; fo wenig, wie ミorgänge in Der aukermenjobliden

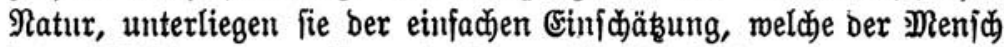

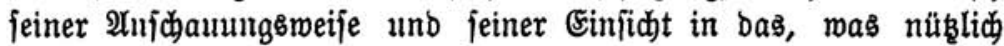
und vernünftig ift, ober was nidjt, verbaultt.

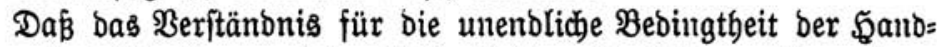
luugen bes Einzelnen unb ber șejamtheit noch nicht zur allgemeinen 2nertenmung gelangt ił, fondern baß man fortfährt, bieje band= lumgen, als bas (srgebnis etraiger, ihnen vorausgehender, freier Entijhlüffe anzujeben, hat babingefübrt, baß bas Strafrecht aud) ber Bölfer, bie auf ber hödjften Sulturitufe ftehelt, nod) it einer meijt ganz zmectwiorigen \&age fïd befinbet,

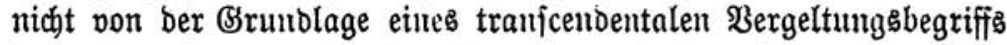
losmacten faum, beffen Durdfiübrung mit ben baburd) erzielten Erfolgen in feltjamem Siegenjaķe fteht, uno deffen ßejeitigung jömerlich in nächjter Beit zu ermarten iit.

Diefe Unberweglidufeit bes Strajrechts iit um fo bemerfens:

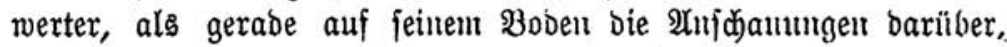
twas Recht ijt und mas nidjt, mehr gewedjielt haben, als auj irgento 


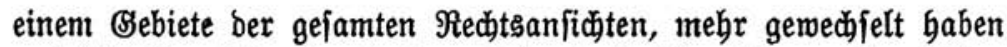
unb fodneller. Seinesimegs ift was beute ftrafbar ift, zu allen Beiten einer Strafe unterworfen gemefen; ebenjo ift aud, was heute ftraf= frei ift, feineşmegs zul allen Zeiten von Strafe verídont gemejert. Der Begriff beffen, was ftrafbar ift, änbert fich in \&aufe ber Jabr= humberte, ja ber Jahrzehnte, und baraus folgt, baß es eine feft=

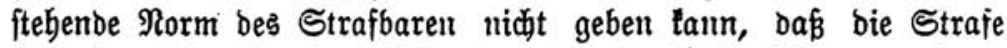

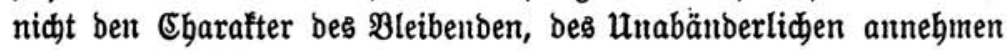
barf unt fann, fonbern baßs bie Begenbantlung ber menidjliden Bemeinfdaft gegen bie Strafthat nidht in einem gleidartigen $\mathfrak{B e r}=$ laängnis gegen alle beftehen muß, bie etmas thun, bas ben Begriff einer als ftrafbar angenommenen \$anolung erfüut, fonbern in einer inbivibuellen Behanblung beffen, ber bie Strafgejebe übertreten hat.

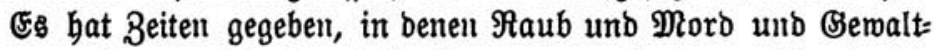
that gegen frauen teinesiwegs ftrafbar maren, fonbern als erlaubt, ja efrenvoll galten. Es hat Zeiten gegeben, beren Sinder erftaunt feil wiurben, wein fie börten, was bei uns alles ftrafbar ift.

24nbrerfeits tennt bie Befdidite Zeiten, in benen ftrafbar war, für was wir heute leine Strafe mehr tennen. $3 \mathfrak{u}$ diejen ড̧anto= lungen gebört bie That ber fogenannten Sererei und Zauberei. Daß̧ fie einft ftrafbar mar, muß aljo nidbt als ein Bemeis von bejonberer $\mathfrak{u n w i f f e n h e i t ~ o b e r ~ g a r ~} \mathfrak{B o s h e i t ~ - ~ a n ~ f i d ~ - ~ a n g e f e b e n ~}$

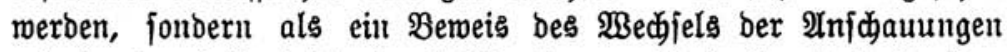
uno ber burch fie bebingten Bejekgebung. Dab fie einft ftrafbar war, ift aber aud) ein Berveis dafür, zu welden Folgen es führt,

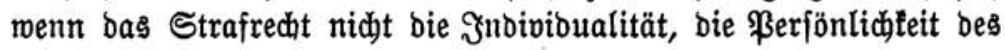
angeblichen Hebelthäters, als feinen eigentlichen BSegenftand anfieht,

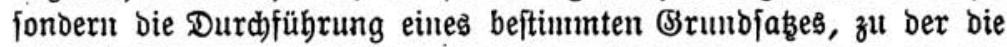
Beidjuldigten nur bas Material fint. Macjoem man bamals ein= mal bie Möglichteit ber Sererei, Der Zauberei anertamut, nadbem man ihre Bsefäbrlidjteit für feffțtebeno angejeben, nachbem man fie zum Berbrechen gemacht, fanden fich) ben verfolgenden (Serwalten Die Sduldigen und ergaben fich ihnen als ftrafbar, ohne Daß man aud) nur eimmal bei ben vielen Taufenten ber Berurteilten in

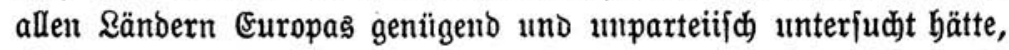
ob benn bie befduldigte ßerjon im ftande gemejen fei, die angeb= licjen $\mathfrak{S e r b r e d ) e n ~ a u s ̧ z u u ̈ b e n . ~}$

Es ift die $\mathfrak{U}$ ebertreibung eines verfebrten ftrafrechtlichen Syftems, was bie (Bejdidte ber Serenprozeffe aud) für bie Strafredhtsmiffen= 


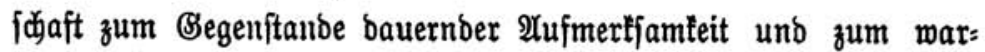
nenden Mertzeiden madjt. Der Brundjak war ba und verlangte bie ihm gebiligrenden Dpfer.

Der Slaube, baß ber Menjd im fande fei, bie Sebeimniffe,

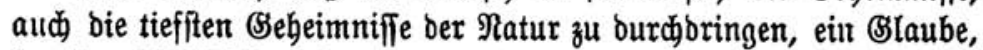

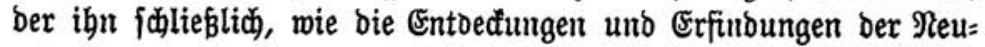
zeit bemeijen, wenigfterts zum Teil nidht getäujht hat, liegt ben uralten Erfdheinungen bei ben veridiebenen Bölfern zu Grunbe, bie man mit bem Ramen ber Zauberei, in vielfacher Form, zu= jammenfabt. Diejenigen, weldie fie betrieben, waren ber 2 unfidt, ber an fich burdaus ridtigen 2 lnfidjt, baß es nur auf bie geeigneten ßerfonen uno Deren zmectmäß̈ige Rräfte anfomme, um bie Ratur

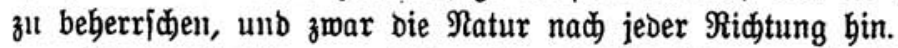

Neuere Froridungen baben es wahrideinlid gemadit, daß̧ bie angebliden Z3auberer, ßriefter und ßeifen ber alten ßölter that= jädliç mebr von ben Sräften ber Natur verítandert, als man bisher altgenommen hat. Währent man bisher vielfach ber थ̂nfidit war, baß̧ wentige Erfahrungen, viel Formenmejen, oft gar Betrug Den Snbalt ibrer Thätigleit abgaben, fäıgt man an einzuleben, Daß jente Reute felbft von Dingen eine Renntnis gebabt baben, Deren

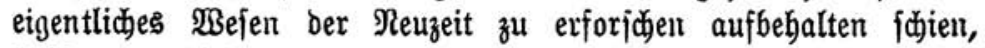
felbft von Eleftriz̧ität, felbit von ber tünftliden Betäubung bei

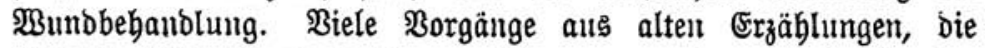
man für $\mathfrak{M a ̈ r c h e n ~ z u ~ h a l t e n ~ g e n e i g t ~ w a r , ~ g e m i n n e n ~ b a o u r d ) ~ e i n e ~}$ ganz anore Beftalt, ganz wie manche eigentümliche Darîtellungen

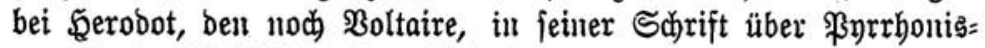

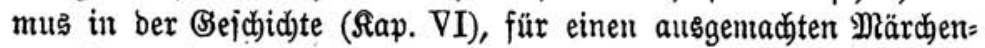
erzäbler bielt, wo er aus fremben Duellen mitteile.

În jenten Beiten badjte beshalb niemand baran, bie jogenamite 3auberei für etwas ftrajbares anzufehen; im Bregenteile waren bie= jentigen, welche fie ausübten, in ibrem Bolfe hodogeebrt, fo - uno vielleidht mehr - wie jeķt große Belehrte uno Rünjtler geegrt werben, was unzählige Berichte aus jenten Beiten auf bas bentlidjite berveifen. Die Jnthaber ber Staatsgewalt ungaben fid) mit ignen,

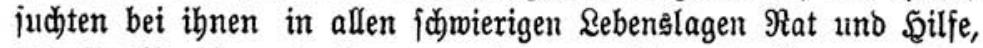
uno igr ßeruf waro ihnen nur dann verbängnişoll, wenn igre Sumjt verjagte, in bem Maß̉e, wie bente etwa ein Staatsmann oder Feloberr leiden mußs, wenn er nidjt leijtet, was von ihm ver= langt miro. 


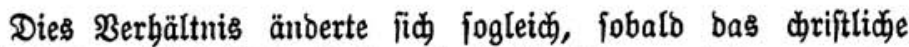
Sirchenfyftem fich befeftigt batte unt in bie Rage verfekt war, ben sampf mit allen ihm feindtidyen, mit allen nicht von igm $a b=$ hängigen ober autgegehenton Mäd)ten zu beginnen ').

शidist Unwiffenbeit war es baher, was bie Sirchengemalt und bie von ihr Sräfte beziehende Staatsgemalt beeinflußte, menn fie anfing, bie jogenaunte Bauberei zu verfolgen, zu ber nod in beit \&änbern mit germanifđjer ßepölferung bie jogenaumte ફ̧ererei trat, eigentlid bas 3aubern burd meiblidbe ßerjonen. Jm Begenteil waren bie maßgebenten Bemalten bavon überzengt, baß man etwas

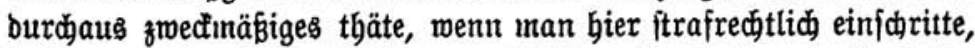
daß̧ man bie tiefften Iiefen menjwlidjer Bosbeit entbect babe, menn man fie aud bort verfolgte, wo fie fid im Bunbe mit andern unbelannten Mäbten, ja mit bem böfen \$rinzip felbft, gegen bie von Sirche unb Staat geleitete Dentidheit verfdmoren habe.

Riemals tounte bieje Beit, bas Mittelalter, ben uriprumg feiner gefamten Sultur aus religiöfen Rebriyftemen verleugnen, und zwar nidit aus felbitgejofjaffenen Rehrifyftemen, fondern aus Sebrinftenten, bie ben Bölfern aus einer Duelle aufgenötigt waren, bie aukerbalb ifrer felbit fló uno nidbt aus igrem eigenen Boben hervorging.

Durch bies 2lufnötigen einer fremtoen Belt: und $2 e b e n s=$ anjobaung feitens einer geiftig viel überlegeneren Macht entwidelte fic) eill \$iderftand, in bem eine Wauptquelle ber lange andauernden geiftigen Sraft ber neueren europäijden ßölfer und bamit eine

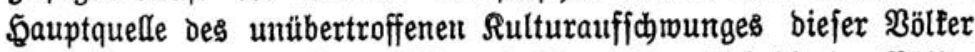
zu finden ift, in bem ibre geiftigen Rräfte, namentlich bie ber $\mathfrak{B o ̈ l t e r}$ germanifden uxiprungs, auf bas äukerfte angefpannt wurben, um jene fremben 2 Infchauungen fich jelbft alzupaffen und fie nadj jich felbft und fich felbft nach ibnen zu bildoen. Ylber nidjt mistoer mar Der Sampf zmifdien ben uriprütrglicjen Rebensanfobauungen ber Bölfer und bem romanij d)=orientalifoden Rehr= und Staatsfyftem

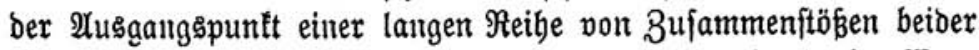
Mächte auf einem Bsebiete, auf bem eine Berftändigung im 2 sege bes Serridermillens niemals ftattinden fonnte, auf bem Bebiete

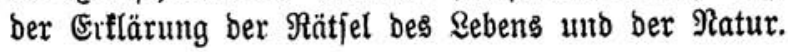

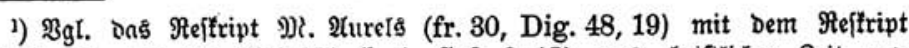
Eonftantin I. vom 23. Daai 321 (1. 4, Cod. 9, 18) aus đuriftlider Beit uno 1. ธ. 6 Daj. 


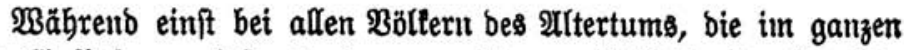
ogne Einfluś, wenigitens obne gewaltjamen Einffub, frember $\mathfrak{A n}_{n}=$ fíbauungen fich entfalteten, biejentigen, bie fich mit biejen Dingen befdäftigten, in bem hoben 2 nifehen ftanben, bas bie Serrichaft über Andern unverftändliche oder unbeherridbare Sräfte ftets ver= Leiht, währent bamals Zauberer, Şeilfünftler mit ifrem ganzen Z3ubebör von überlieferter Weisheit ber allgemeinen ftaumenden Bemutberung fich erfreuten, fah bie Staat uno \&eben beherrifienbe

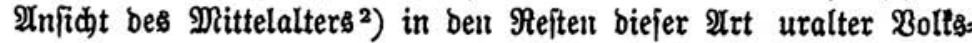
weisheit einen natürlichen Freino, einen Feind, Deffen Sdöpfer nad ihrer Denfweife nur ber Bruno alles Böjen, ber Teufel, feit fonnte, bem fie gebörten uidit ihr an und nur fie und was oon igr ausiging, entfaumte bem Sidjt unt ber \$3abrbeit.

Schon Sarl I. ließs im Jabre 785 zu ßaberborn vour ber Synobe befdließ̧en, baß̧, wer vom Teufel verblendet, nad æBeife ber Seiben glaube, es jei jemand eine \$ere, - mit bem Tobe bejtraft werben jolle. Mit Deutlidjteit zeigt biejer \$ejdlußs, baß ber Bolfsglauben, bas jogenannte Seibentun, es war, in bem bie herridende Bsemalt bas Strafmürbige fallo, daß es bie Refte ber Boltsantfdauung marent, Denent fie einen Utriprung aus bem Böjen, aus Dem Telfel, zujळrieb und Srieg anfünoigte.

Die Sirche igrerjeits mufte umjomebr auf biefem Standpuntte verbarren, als fie felbft fich eine Madjt über bie Natur zufdorieb und auf biejem (Sebiete eine ähuliçe Thätigteit, bie aus einer nicht von ihr abgeleiteten Duelle entiprang, nidyf bulden tonnte, - nidjt nur eine foldhe Thätigfeit, fonoern aud ben (Slauben an bie Miog=

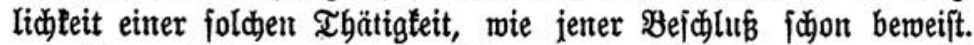

Iber nidjt mur hierin, niđt mur im Mideritande gegen bie

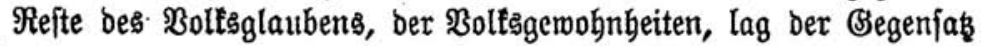
bes volt ber Rirdje beberridten Mittelalters gegen Zauberei und Sererei begrünbet. Sondern, inbem bie Sirche fid im Befiz̧̨e un=

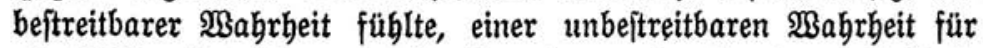
alle Bebiete bes Lebents, wohin fie gelangen tounte, einer unbeftreit=

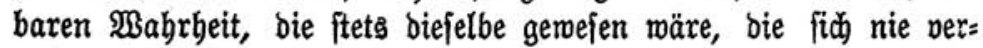
ändert babe, fo bildete fid für bie herridende 2rujidit bes Mittel= alters ber Bebante aus, baß

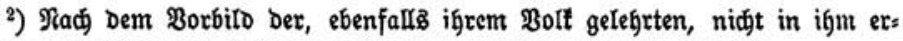

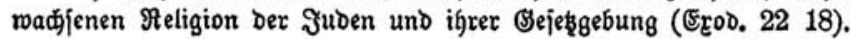


aud f̣tets für wahr gebalten fei, eine unveränberlidbe, emige, gött= liche $\mathfrak{B a b r b e i t ~ f e i n ~ m u ̈ f f e . ~}$

Jebe Sritif, jeder 3meifel murbe fern gebalten. Was nidjt in bas Syjtem einzuorbnen war, eridien nidht als Brunblage einer \$rüfung, fonbern als unwabrbeit, als zurüđżmeifen, als zut ver= nidbten, und fei es mit ber größiten 2 ufbietung aller Rräfte. Berabe das freie Spiel ber Mleinungsäuß̧erung, weld)es die Begenwart für bie widjtigfte Mradyt zur Erforidung einer annebmbaren $\mathfrak{B a b r b e i t}$ erfannt hat, war bas, was bie mittelalterliche $\mathfrak{B e l t a n j i d a u u n g ~ n i d t ~}$ buldete, nur in beftimmtent Brenzen buldete, jebenfalls nie zur Selbitänbigteit heranmadjien ließs.

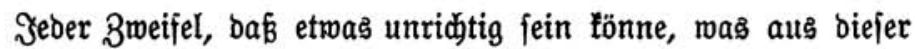
Weltanidauutg beraus gelehrt unto gethan wurbe, was aus ihr beraus gelebrt und gethan worben war, waro mit vollem Bemußst=

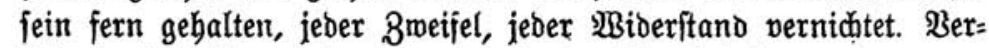
nidłtet, nämlid) entweber unterorüdt ober fein Urbeber getötet, oder

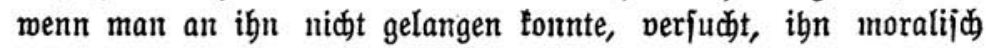
in ber Bolfsmeinung zll töten, indent mall ign für ein Sino, einen Freund des Tentiels ertlärte. Das ßerfabrent gegen Saijer Friebrid) II., von Dem bie blutigften Strafgefekse gegen $\mathfrak{A b f a l l}$ pon ber Rirche ausgegangen waren, megen feiner perjönliçen Stellung zut ibrer Rehre, läßst einen \$liof in bies ßerfabren thun; jelbft ber B̧bibelline Dante feķt ign in bie Gölle (Inferno. c. 10), zmeifellos in Uteber=

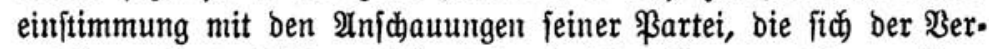
urteilung ibres einftigen Füürers burch feine Bsegner nidyt zu wiber= fezen vermochte, ałs gaulz gerecht und fachgemäß.

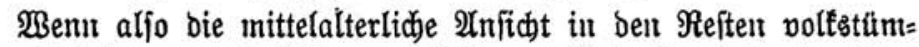
licher Erfahrung uno Rebensmeisheit unt Raturfenntnis in jeber felbitändigen 2 nficht barüber einen Feint $\{a \zeta$, wenn fie biejen Feind als vom Teufel ausgebent, als ben Teufel jelbit erfannte, fo war bamit ihr Sdjidjal entjdieden uno all' igre Teilhaber ber ßer= nidtung ankeimgegebent.

Der Bedanfe, den bie Neuzeit für ben 2 thgangspuntt alles mabren Wiffelts bält, - Dấ es Dinge giebt, bie miffeltsmert find, bie man aber nidjt meiß̄, fam in jenen Beiten nidbt zur Beltunt; man mußste, nahu man an, alles, was man miffen fomte, uno was man nidyt wuste, erjoien entweder überflüfiity ober verboten zu wiffen, aljo bas Streben barnac) verwerflic) ober ftrafbar, von 
Dem Bsegeniak beffen auggegangen, ber bem Menjळen alles gezeigt und offenbart, was ihm zu wiffen nötig ober nüb̨lid, ben Teufel. Nidht allen faum fdyon bamals bieje Rebre genügt babent, benn

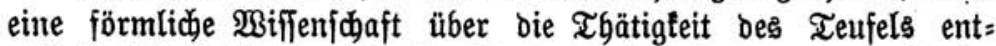

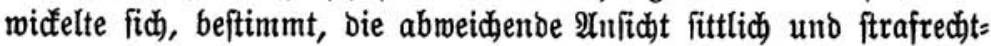

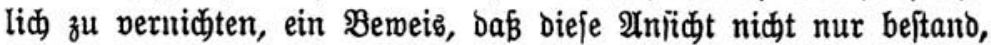
fonbern aud Fortichritte zu maden brobte.

2uf biefe Weife warb bie F̧urdt, gegen bie Rebre Der Sirdhe veritopen zu baben, eine bauernbe, und aus biejer Furdt floß eine ftete Duelle ber Macht und bes Einfluffes ber Rirde auf bie Be: völferung. Sie bejand fich 'it einer fteten 2lufregung, baß ber Teufel nidht vout ihrer Seele ober ber Seele ihrer Angebörigen und (B)emeindegenoifen Befiz ergriffen babe, in einer ftetell Furdit, Daß̧ ber Teufel fid ihnen, ibren Angebörigen, ibren Bsemeinde= genoffen nabe. Dem 2 uperorbentlichen, bas unerflärbar fibien, wurbe nidyt meiter nadidgeforidst. (Sejprochen ober getbalt, wurbe es bent Tenfel zugeidriebelt, jei es, baß er unmittelbar eingemirtt,

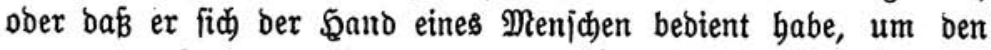
georoneten Bang ber Ratur zu ftören.

Je mebr bie Rehre ber Rirdje - und fie war bamals, was man nie vergeffen muß, bie einzige lehrende Stelle - in bie Be: völferung einorang, befto größer murbe bie furdst vor ber \$irt: famfeit ber bie Menjøent luberall und jeberzeit umgebenden böfen, unfidtbaren (Sewalten. Diefe Furdit wurbe zu einer Wauptfraft, Das (Bebäude bes priefterlicjen Staates zufammenzubalten, Denn jebes Wort, jeoe That gegen biejen Stant, aud nur im entfern= teften Simue, war geeignet, ben Urbeber bürgerlid und förperlid zu vernichtert.

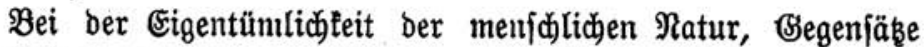
aufzujuchen, mit ibnen zu fämpfer, unterliegt es feinem 3meifel, daß́ dies Sach) und Rechtsenverbältnis, wie es Millionen im Bebor=

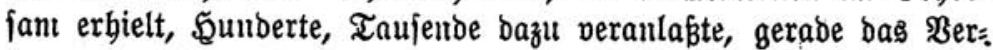
botene zu thun, fich bem, was bie berrichende Rebre ben Teufel naunte, zu ergeben uno fid mit ihm zu verbünton, ja felbit zu glauben, baß fie mit bem Teufel verbünbet wären und ihın, felbft

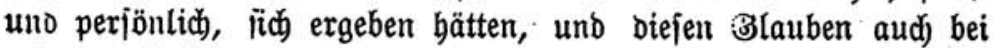
andern möglid) zu machen und zu erbalten.

Diejer ßorgang ift ein jo auferorbentlicher uidjt, wenn man bedenft, wie vielfach die Rebre ber Sirdje von ber inmbolijdjen \$e: 
zeidynung eines Begenftandes bazu fortgejdritten ift, biefe : Bezeid)=

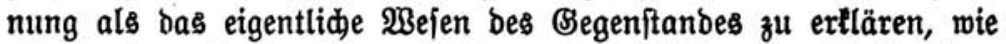
oft ein Begenftand, ber zunädfit nur bem Bilbe, ber Sirche einer

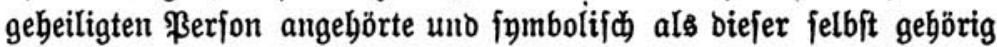
bezeidnet wurbe, follieplid) als ihr Eigentum thatjäblid an= gefehen wurbe.

Die Berfolgung ber bem Teufel 2 ngebörigen förectte taufente von bem Bünonis mit în $a b$ uno fübrte igm, mie erfabrungs: gemäß alle öffentlichen ober alle phantafieerregenben geheimen $D_{e}=$ ftrafungen blutiger $\mathfrak{A} r \mathrm{rt}$, hunberte zu.

Jebod fdeint in ber erften Zeit bes Mittelalters, bis etwa zum 13. Jahrhumbert, eine Berfolgung berjenigen, bie fid Dem

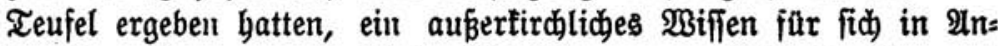
fprud nahmen, zur Zahl ber Bevölterung verbältnis̄mäßig nur felten gemejen zu fein; nur einzelne ßerjonen unterlagen in biejer Beit ber Beftrafung, ojt unter bem Wiberftande ibrer Bemeinde: genoffen gegen bie ftrafente Bewalt.

Die Sacblage änoerte fich aber im 13. Sabrhunbert, in weldhem faft zugleidy in Sübfranfreich, in Norbdeutjolano, in Rorbitalien, in Englano, in Dänemarf bie vorbandenen nationalen ober ftaat= liçen Sräfte fich zum lek̨ten Berzmeiflungšfampfe gegen bie ftei. genden 2 nnforberungen ber Befteuropa beherridjenden Sirche maff= neten, und bie Rirche ihrerjeits iłhe Bläubigen gegen ihre Feinbe, als gegen Teufelsanbeter, Režer, Zauberer, Weren in bie $\mathfrak{\text { Baffen }}$

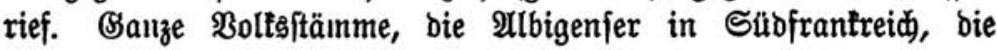

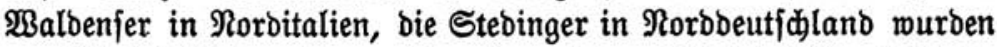
vernidytet, teils in offenem Rampfe auf bem Sd)ladtfelde, teils im Strafverfabren auf bem Sdeiterbaufen, ein reuer :erweis bafür, baß̧ bie Macht des mahren Sottes höber ift als bie bes Teufels, menu fie ihn auch eine Zeitlang gewähren läßjt.

(Banz unbeftreitbar hat bie Bejduldigung eines Bünoniffes mit bem Teufel, bas man biefen Feindent ber Rirche zur Raft legte, ihre Saupturjadje in ber 2 bjonberung von bem Esottesbienft, von ben Bebräudjen und ßerjonen ber Rirche. Wenn fie nidt an= betetelt, wo bie Sirche Bott verebrte, melın fie ihre Bebräudje nicht

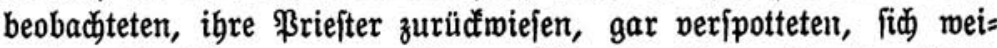

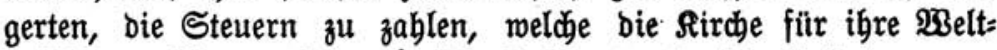
politif von innen erbeben wollte, fo tonnte, ba bie 2 nnforberungen Der Sirche voll Ssott ausigingen, bas Thun biejer Siez̧er, Zauberer 
und Seren nur vom Teufel eingegebent fein, und in igren $3^{\mathfrak{u}=}$ fammentünften nidht Bott, fonbern ber Teufel verebrt, nicht (S)utes und Wabres bort betrieben merben, fondern Berbrechen und \&üge

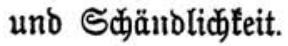

Unter biefem Zeidjen gewann man Begner und Feinbe ber

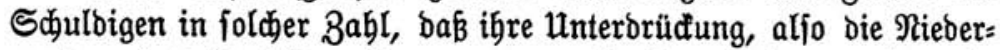
werfung bes Teufels, gelang, wenn aud oft nach langem Saunpfe, in Sübfrantreid nach 20 J̧ahren.

Die eigentliche Stantaggemalt ftand bei biefen Unternebmungen antsnabmolos auf jeiten ber Sirche, benn fie batte vom Siege ber Sirche, als ber berridenden Madt, mur zul geminnen, wie es benn überhaupt während bes gejamten Mittelalters, bie Zeit ber beut=

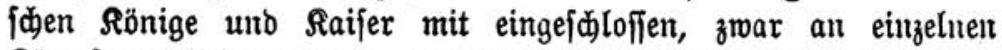
Sämpfen zmifdell Staats= uno Sirdengewalt nie gefeblt bat, aber fein einzziger Staatslenfer, bis auj Sönig জeinric VIII. von Enı= lanb, jemals auf ben Bebanfen gefommen ift, mit ber von Bott ftammenton \&eitung ber Sirche grunbjäbllid fich auscinander зu jeşen.

Mit biejem Siege ber Sircje war jeder Mideritant ganzer Maffen gegen ibre 2ulleinberriłaft über bie gefamte geiftige Rultur

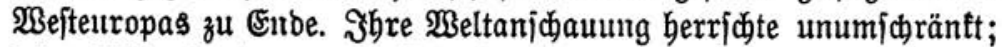
jeber Wiberftand war ausfididstos und verfiel ber untausbleiblidjen Strafe, umjomebr, als er unr vereinzelt auftrat. Die aus bem

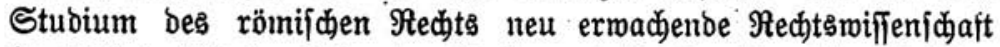
bemädtigte fich ber Rebre der Rirche uno bemies ihrerjeits bie ßer= nichtung ber Teujelsanbeter, Bauberer und Seren als burch bas bürgerliche Bejęz geboten.

Die Rirche hatte bie Racbirtungen ber eigentümlidjen Rultur ber einzelnen wefteuropäifden $\mathfrak{B o ̈ l f e r}$ im Zujammenhange über= wunden. Sie war ibrer unbeftritten Serr gemorben; aus jenen Siräftent hatte fie niduts mebr zu fürd̆tent.

2Xtber es batte fich ein netter Feint erhoben, bie feit bem

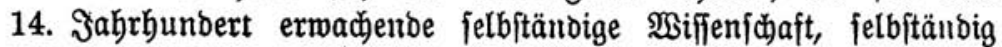
von ber Regre ber Sirche, zunächft allerbings nur neben ihr, oft fogar unter ihrem uno ihrer Şäupter Schus; biejen Feino, Deffen Madjt bisher ftets gemadjen ift, hat fie nicht wieber untermerfen łönnen. Die Sirche börte allmäblid auf, allein an ber Spik̨e ber geiftigen Entwictlung Wefteuropas zu ftehen; fie maro eine politijche Macht und es begann eine Sultur aukerbalb ber Bebiete, die fie 


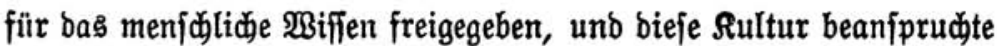
fdjlieblid, ber ber Sirche überiegen zu fein.

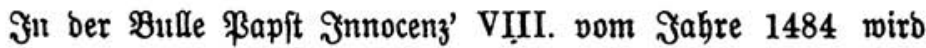

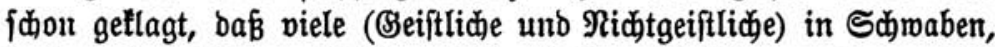
in ben Erzbiztümern Mainz, Söln, Trier, Salzburg, Bremen fid Der Beftrafung ber Zauberer und ફeren, beren gemeingefäbrlides Treiben bie sulle eingebend fojilbert, wiberjekzen,

"bie mehr wiffen mollen, als man barf",

aljo gerabezu bie Rebre ber Sirche in biejem \$untte für unridjtig ertlärten.

Mit biefer \$ulle raffte fich bie herridende (Bemalt zum nettent Berniditungstampf gegen \$erell: uno Zauberwejen auf, ber, na= mentlid in Deutidland, hunderte zur Beftrafung lieferte und um jo ergiebiger war, als aud biejenigen nad) biejem neuen Bseję bejtraft merben follten, bie nidjt an Şeren und Zauberer glaubtelt.

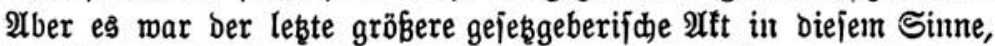
ben bie alte Sirde unternabm. Sie mar, nad faum 40 Jabren, felbft in bie Rage verjeķt, um ihr Befteben zu fämpfen, uno fie ging aus biefem Rampfe, nicht fomohl an äuß̉ern Madjtgrunolagen geidumäd)t hervor, als vielmehr um ihr ફ̧aupttampfmittel, nämlid̆ bie, in \$efteuropa allgemein anertannte, 2Uleinberedtigung, bie

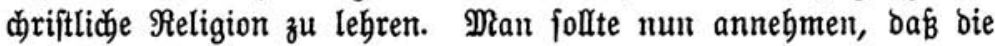
neu entítandenen Religionsegemeinidjaften bie ganze ftrafrecttidide Berfolgung Der 2lnfidten und ûberzeugungen, bie einft ibre eigene Entfitefung vielfach gebindert uno bejdräntt hatte, ibrerjeits be: endigt hätte.

2rber bies war nidt ber frall.

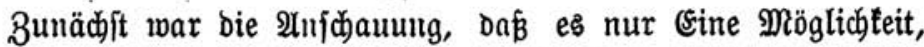
nur Eine Quelle gebe, bie driftlidje Religion zu vertündelt, fo tief in bas Berwußtfein ber Theologen und aud bes Bolffs eingeorungen,

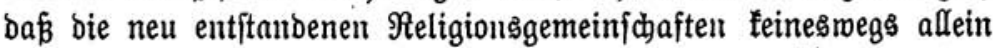
mit ber Forberung ibrer 2lnerfentuung auftreten burften, - bas

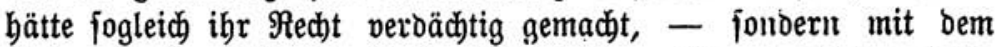

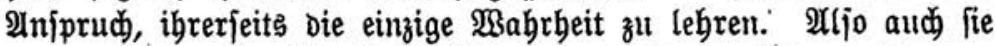
mußten nun abweidjende 2 Anfidten von igrer Lebre als vom Teufel entítanden erflären und nabmen zunäddft ber alten Sirche ganzes Rebrinjtem, menigitens über bie Brenzen menjwlidjen Er: felutens, auj. 
Ferner gaben bie netien Religionsgemeinjofaften, bie unter Dem Schuke ober mit bem (sinfluß ber bürgerlichen, ber ftaatlidjen Bewalten - teils als joldjer, teils ibrer J̧nhaber - eingeridjtet waren, nach und nach bie gefamte geiftlidje Regierungs= und Straf= gewalt an bie Staatsbeböroen ab und ber Staat trat fomit, burch

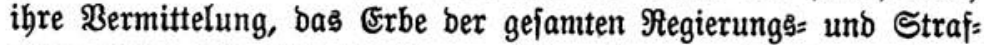
geiesgebung ber alten Sirche an, aljo aud gegen Zauberer, Weren und Teufelesbünoniffie; man bebiente fich ganz felbftveritänolich ihrer Bejeşe, auj vielen Bebieten des \$rivatredits now bis in bie neuefte Beit.

Die Berfolgung bes Seren=, Zauber= uno Teufelsmejens trat bamit in eine nette, die leşte Entwiđđlung ein, bie. Berfolgung burdh bie Beridhte ber Staaten.

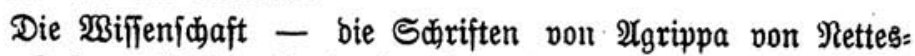
heim, Johann ßeier, Reginald Stot, Bobelmann, Montaigne -

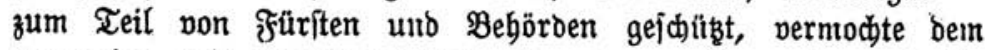
gegentiber nicht zur Қerrichaft zu gelangent, um jo weniger, als bie Stimmen gèteilt maren und fich mindeftens ebenfo viel S'egner jeder Neuterung fanden, unter ihnen Rönig গ̧alob I. von Broß̧britannien unt Jrtano, Der in feinem Werfe über Dämonenlebre zwar be: hauptet:

"no die Unmiffenbeit ber Pesijuen am biđfiten ift, ba ift aud bie Uuıveríbämtbeit bes Teufels am größten",

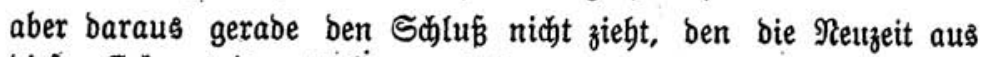
biefer Erfenntnis entnebinen mürbe.

Dennod war bamit, baß bas verfolgte ßerbrechen in einer

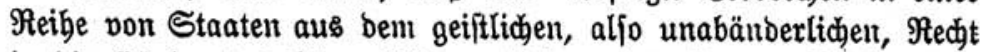
in bie ફ̧änbe des bürgerlidjen, aljo veränberbaren, Rechte einer

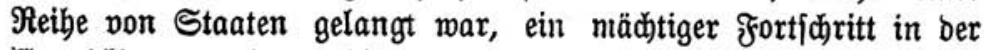

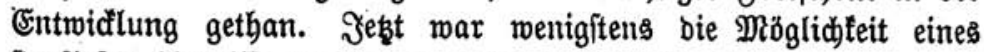
ftraflojen 2 ngriffs gegen bas ganze ßerfabren gegeben.

Die bürgerliche BSefekgebung freilich, foviel ihrer Teilbaber felbft in jener Beit an feiner Berechtigung gezweifelt baben mögen, buldete bie Fortjęung bes Berfahrens; foweit mar ber Z3meifel

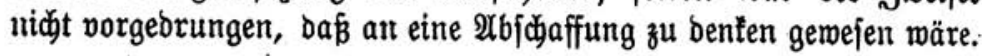

Smmerhin ift in bem bebeutendften ber Strafgejetzbüdjer jener 3eit, Dem Ratjer Rarls $V$. von 1530 und 1532, eine gemiffe Mit= Derung nidyt zu verfennen. 


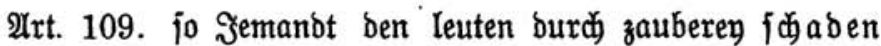
ober naditeyl zufügt, foll man frafen vom lebell zum tobt,

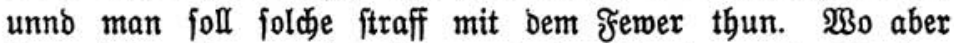
Jemandt zauberey gebraudit, uno bamit niemand fobaben gethan batt, foll fonit geftrafft werben, nad gelegenthent ober fach, barum bie urtheyler rabts gebrauden follen.

Deutlid ift bier zu erfennen, baß ber zugefügte Schaben burd

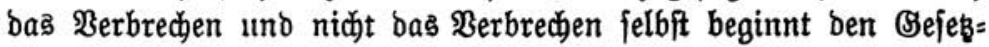
geber zu beftimmen.

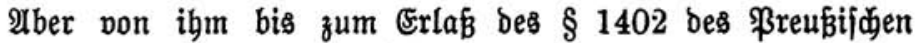
Irflgem. Sanbredts vom 3. Februar 1794 II, 20:

Seute, bie burd betrüglide Bauleleien, als Bsolbmad̄er,

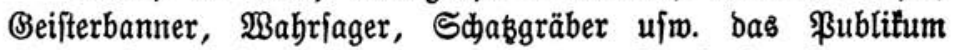
bintergehen, Gaben, aufier ber ordinären Strafe bes Betruges, 3uththaueftrafe auf fects Monate bis Ein Jabr uno offentliche 2lueftellung vermirtt,

und ber $\S 86$ besfelben seejeşbuct) I, 9:

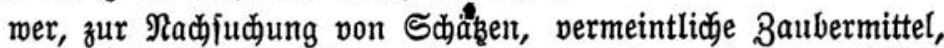
aud Beifterbannell, Eitieren Berftorbener ober anorer bergleidyen Baufeleien, es fei aus Betrug ober 2rberglauben, fich bebient, ber verliert, aufer ber fonit fdon verwirtten Strafe, jein Ât= recht auf ben etwa zufäliger Weife wirflid gefunbenten Sdaką, als ber gefę̧geberifichen Aluffaffung ber Zauberei unt Şererei als

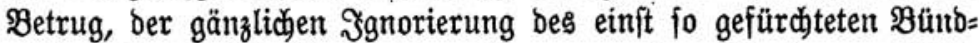
niffes mit bem Ieufel, in einem hervorragenden Staate von $\mathfrak{B}_{\text {eft }}=$ europa, liegt eine Beit von noc) nicht breibundert Jabren, aber bieje Beit ift eine Blütezeit ber Berfolgung ber \$exerei, Zauberei, bes Teufelsbünoniffes, bes Slaubens an ihre verberbliche \$irfung.

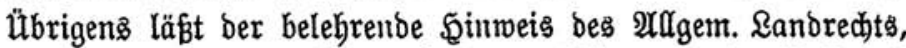
baß man einell, beim Zaubern gefmbenen Sdak nur burdib Bufall

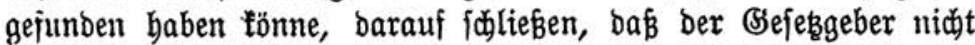
alfeitiger übereinftimmung gemiß war.

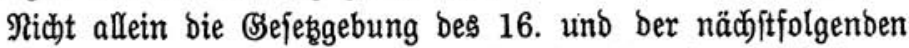
zmei Jabrbunberte nabm Stellung zu Sumften ber Strafperfolgung jener Berbrechen; bas Beridjtsmejen biejer Zeit that alles, um bie bejtehenden Befese mit unnachfichtlicher Schärfe zur 2lnmentung zut bringen und exit bierin, in ber 2Ynmentung jenter Bejebe, lag Der $\Re$ uf begrünbet; Den bie bamalige Zeit ermorben. 


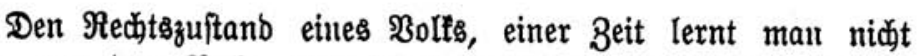

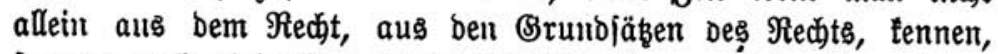

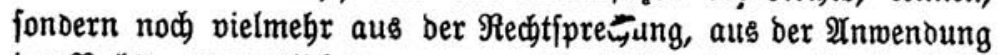
bes Rechts, namentlich Dann, menn bie Befeßzgebung nidht fo thätig ift, wie in unjern $\mathfrak{T a g e n .}$

Damals tannte man fein $\Re$ echtsmittel. Wer verurteilt war, ber war fogleid) rechtêträftig verurteilt, wurbe oftmals aud fogleid, zur Bolffreçung, felbft ber Tobesitrafe, geführt. Nur, wemn ber

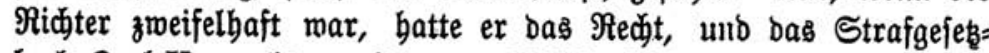

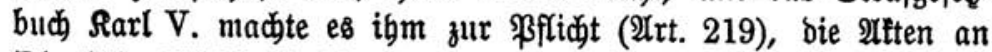

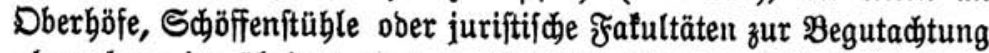
abzugeben; im übrigen war er alleiniger জ̧err ber Thatbeurteilung

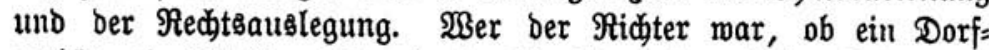
geridit, ein Stabtgeridt, ob ein Mragiftrat einer freien Reidsftabt Doer ein Beridbtsbalter eines Ritterguts, war gleidjgiltig - er

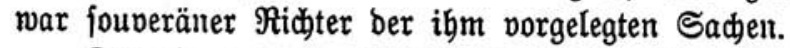

Damals gab es feine Juftizaufiidyt. Man tannte allerbings

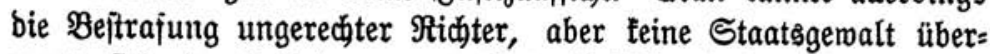
zeugte fich, burch ftete, unauggefește 2 Yufficht, baßs auch alle ftraf=

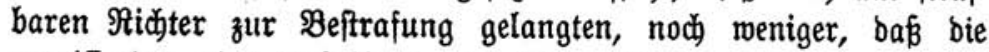
unmiffenden ober unfähigen vom 2 mt entfernt wurben, am aller= wentigften, baß bie vorliegenden Sachent täglid) oronungşmästg be= arbeitet würben.

Diefe 2 Ufficht und Rechtemmittel wären um fo bringenter not:

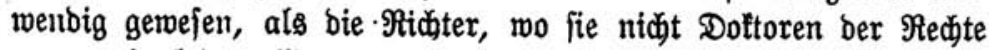
waren, in teiner \$eife burd) beftintmten Rehrgang zu ibrem $24 m t$ vorgebilbet waren, fonbern bie 2Unftellung ihnen bie ßefäbigung gab. Man lebte in jener Zुwifdenzeit zwijden ben Jabrhunderten,

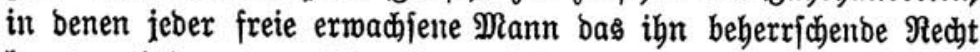
launte und benen, wo feime Senutnis das Ergebnis eines, ein ganzes Menffhenleben fortgejeşten, eingehendften Stubiums ift.

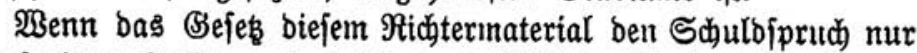

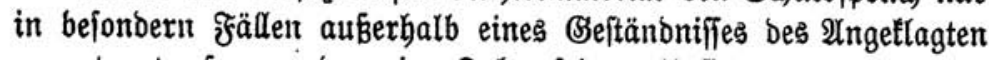
anvertraute, fo war bas eine Folge feiner Einficht in bie ßerbält= niffe; aber bie barin liegente ßorficht murbe burd) bie Anmendung in bas Begenteil, nämlich Miß̧behanolung ber Bejchuldoigten, ver=

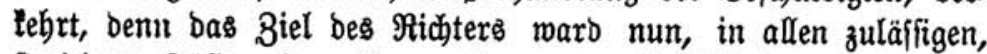
ftreitigen Fällen ben Reugnendent zum Beftänonis zu bringen,

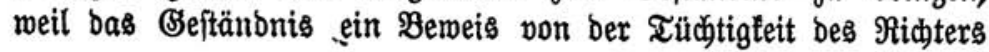


war, wie bente eine umfaffende ftrafredtlidje Einfidyt und Er: fabrung.

Dem gegenüber reidten auch bie genauejten, forgfältigiten Boridriften Der Bejęzgebung, um zu verhindern, baß voreilig ein Beftänonis erzmutgen, baß bem erzmungenen Beftäntonis voreilig geglaubt merbe, nidft aus. Irt. 44 bes Befeezes Rarl V. fagt:

fo Jemandt fich crheut, andere Menidjen Zauberey zu lernen, ober Semandt zu bezaubern bebrohet und bem Bebrobten ber=

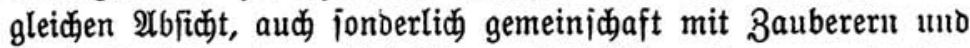
3auberin hat, ober mit foldent verbechtlichen biligen, geberben, worten uno weijen, umbgeht, bie zauberey auff fid tragen, uno bie felbig perjon desjelben funit auch berechtigt, bes gibt enn reblich allzengung ber zauberey, und genugiam uriach zul peill= licjer frage.

Man verfugr aljo in Deutjhland bem beftehenten Recht ge= mäв, wenu mau bie Frolter gegen bie Befd)uldigten zur Ulnuventung brachte. Sdjonung ber Sđulbigen hatte ber Selekgeber teinenfalls gewollt, zumal baffelbe Bejeşbuch ausbrüdtich autoronet, baß ber

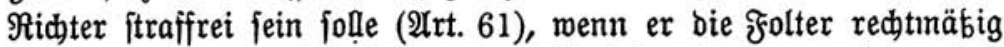
gebraudjt uno ber Befolterte als unjduldoig erfannt morben wäre.

Enolid, verbient nidjt unerwäbnt zu bleiben, baß bie Befol=

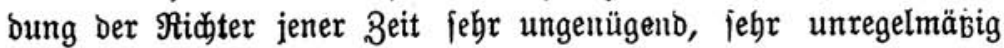
angeoronet war, daß̧ fie zum groken Teil aus ben Strajgeldern, bie aus bent Bermögen ber Berurteilten bejtritten wurbe; wer freifprad), exlitt einen Einnahmeverlujt. Maut befand fich aud bier

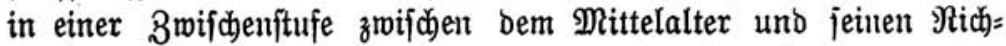
tern aus Brunbbefiţern uno andern unabbängigen Reuten und ber Reuzeit mit ibren Beridjten, die mit bejolbeten Beainten be= jeķt fino.

Faß̧t man alle biefe ßerbältniffe in ein જild zufammen, fo ift bie Erideinung natürlich), Daß̧ feine Beit mäbreno eines jo langen Berlaufs, fo wieberballt von Rlagen über bie Untgerechtigteit,

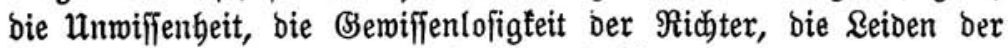
Inneflagten. Sie waren vorbantoen, felbft menn man aunebmen

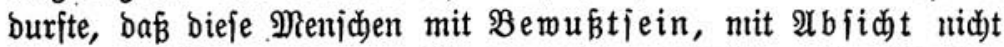
in bem Make vorgingen. Die Darftellung ber ßrozeffe gibt aber oft ben Einbruct, als ob eine Meute von Raubtieren gegen bie

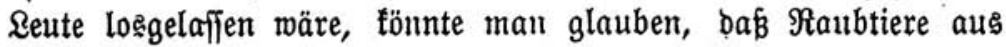


Wohlgefallen am Martern töten; namentlid gegen Frauen wurbe oft berartig verfabren, baß ber ßerbadjt entfteht, es haben bier Snoiviouen bas gejudt und gefunden, was fie beute in ben fo: genamuten Ruftmorben fuchen und finden: Serbinbung von Bolluft mit wilbefter Blutgier.

Xber aud hier fübrte bas übermaß bas Entoe bes ganzen Thuns herbei.

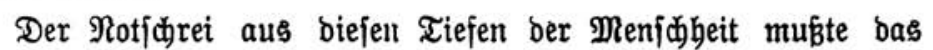
Serz ergreifen, wenn nicht Der Beritaub zu Şilfe fam. Frriebrich von Spee, einft Beidhtwater vieler Berurteilten, in feiner Cautio Criminalis (1631), Egriftian Thomafius in feinen Thejen vom Jabre 1701 ergriffen bas \$ort. Einfidutsoolle F̈ürften, zuterft Ehri= ftine Sönigin von Sdmeden, bam bie Serricher aus bem ફ̧auje Sobenzollern fahentten ignen Behör. Unnbetümmert um \$olts:

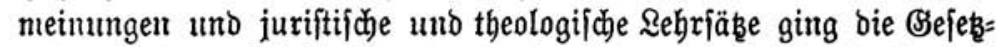
gebung mit ber 2̂ufhebung Des Berfabrens gegen Zauberei, \$ererei,

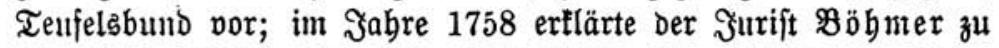
Franffurt (Doer), báb ber serenglaube ber Beradtung preis: gegeben jei.

Die ntaßggebenden Madiftellen fürdyteten meder Zauberei, noch

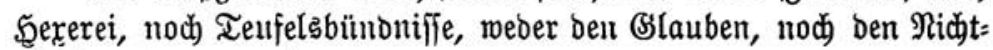
glauben barau mebr.

\$reußen fdaffte das ßerjabren bagegen im Jabre 1728 ab, nadjoent fidon 1701 bie eriten Maßregeln getroffen waren, 1738 Sdjottlanto und Englanto, 1766 D̈iterreid), 1779 Sibmeden, 1776 \$olen, 1821 Эrlanto; bie lekzten Werentiprozeffe merben berichtet aus bem Jahre 1731 in Franfreidh, 1724 in Sicilien, 1775 in Sempten, als Dem leşten auf beutjder Eroe, 1782 in Blarus, 1874 in Merifo.

Der Sslaube an Serell, an Zaubern bauert bem ungeaditet im Bolfe und it ben ভdyriften von Theologen ber driftlidien Sonfeffitonen fort, bie fid) nidst entid)lieben fönnen, mit Rehren ber Bergangenbeit zu brechent. Ûm jo mebr fteht unwiberleglich feft,

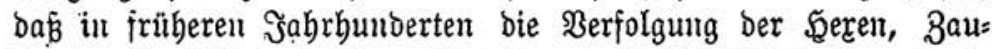
berer, Tenfelšverbünbeten fein Erzeugnis des Betruges, fei es, woher er fomme, gewejen ift, fonbern, daß bie meiteuropäifde Dienijheit von ihrer Berechtigung überzentat war, und bie berr= fchenden (Semalten von ibrer (Befäbrlid)feit, fo viel Täufdurg, 
vielleidyt bewußste Täufd)ung im einzelnen Falle aud vorbanben

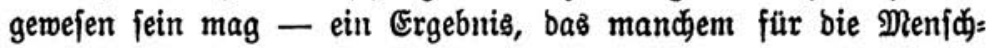
heit weniger ebrenvoll bebüntent mag, als wenn Betrug bie ein= fache Utriadje gemefen wäre; aber weniger ehrenvoll ober uidut, es beweift, wohin bie Meniden gelangen, went fie nidits fitört auf bem Bege nach jebem Ziel, nac bem fie ein vorbunbener Slaube weift. 